\title{
Comparison between B-mode ultrasonography and contrast-enhanced ultrasonography for the surveillance of early stage pancreatic cancer: a retrospective study
}

\author{
Jin $\mathrm{Xu}^{1{ }^{1 *}}$, Meng Zhang ${ }^{1 *}$, Gang Cheng ${ }^{2}$ \\ ${ }^{1}$ Department of Medical Ultrasonics, the Affiliated Hospital of Jiangnan University, Wuxi, China; ${ }^{2}$ Department of Medical Ultrasonics, the Affiliated \\ Wuxi People's Hospital of Nanjing Medical University, Wuxi, China \\ Contributions: (I) Conception and design: All authors; (II) Administrative support: G Cheng; (III) Provision of study materials or patients: J Xu, M \\ Zhang; (IV) Collection and assembly of data: J Xu, M Zhang; (V) Data analysis and interpretation: J Xu, M Zhang; (VI) Manuscript writing: All \\ authors; (VII) Final approval of manuscript: All authors. \\ \#These authors contributed equally to this work. \\ Correspondence to: Gang Cheng. Department of Medical Ultrasonics, the Affiliated Wuxi People's Hospital of Nanjing Medical University, Wuxi, \\ China. Email: 653762580@qq.com.
}

Background: Contrast-enhanced ultrasonography (CE-US) brings a higher signal-to-noise ratio and a higher sensitivity for slow flow than traditional B-mode ultrasonography (US). However, it remains unclear whether CE-US is also superior to B-mode US in detecting early-stage pancreatic cancer (PC).

Methods: This was a retrospective study enrolling patients suspected of pancreatic insufficiency between June 2015 and December 2019. Enrolled patients successively received B-mode US and CE-US examinations, and some their demographic and clinical data were collected. The diagnostic capacity of the two examinations was calculated and receiver operating characteristic (ROC) curves was used to compare the area under the curve (AUC). A subgroup analysis was performed to explore the effects of tumor size on the diagnostic accuracy of B-mode US and CE-US

Results: There were 128 patients enrolled in this study; 74 patients were diagnosed as early-stage PC patients and the remaining 54 were diagnosed with benign pancreatic lesions. The mean size of the PC was $17.8 \pm 4.9 \mathrm{~mm}$. The results revealed that 68 of the $74 \mathrm{PC}$ patients were correctly diagnosed by CE-US, and all 54 patients with benign pancreatic lesions were also correctly diagnosed. Meanwhile, only 55 of the 74 PC patients and 50 of the 54 patients with benign pancreatic lesions were diagnosed correctly using B-mode US. The ROC curve showed that the AUCs of CE-US and B-mode US were 0.959 and 0.835 , respectively. According to the subgroup analysis, CE-US exhibited better accuracy than B-mode US for smaller tumors (size $<20 \mathrm{~mm}, \mathrm{P}=0.002$; size $<10 \mathrm{~mm}, \mathrm{P}=0.043$; size $<5 \mathrm{~mm}, \mathrm{P}=0.025$ ).

Conclusions: CE-US was clearly superior to the conventional B-mode US in detecting early-stage PC, especially smaller sized PC.

Keywords: B-mode ultrasonography (B-mode US); contrast-enhanced ultrasonography (CE-US); surveillance; early stage pancreatic adenocarcinoma

Submitted Sep 14, 2020. Accepted for publication Oct 14, 2020.

doi: 10.21037/jgo-20-412

View this article at: http://dx.doi.org/10.21037/jgo-20-412 


\section{Introduction}

The prognosis of patients with pancreatic cancer (PC) is usually poor, with a 5 -year survival rate of less than $10 \%(1,2)$. Complete surgical resection could provide PC patients a relatively satisfying prognosis (3). However, most PC patients have no specific symptoms in the early stages, and thus, often miss optimal opportunities for treatment. The majority of PC patients require chemotherapy treatment, which can only provide a minor improvement (4). The earliest possible diagnosis and treatment of PC is key to improving the prognosis of PC patients.

To date, due to the lack of specific serological biomarkers, the early diagnosis of PC mainly depends on imaging examination. Several imaging modalities have been utilized for the diagnosis of PC, including ultrasonography (US), computed tomography (CT), magnetic resonance imaging (MRI), and endoscopic ultrasonography (EUS) (5-7). Abdominal B-mode US is a minimally invasive and convenient imaging examination to detect PC. However, it has been reported that B-mode US has a sufficiently high sensitivity but a low specificity (8), as some other benign lesions may show similar US images similar to PC. Therefore, there is currently a pressing need for alternative examination methods to further confirm the diagnosis of PC. Some invasive procedures, such as fine needle aspiration biopsy and endoscopic retrograde cholangiopancreatography (ERCP), could provide more effective diagnostic information (1). However, such procedures may potentially lead to severe complications.

Contrast-enhanced US (CE-US) has been proposed for several decades to compensate for the inherent limitations of traditional B-mode US. With the administration of an ultrasonographic contrast agent, CE-US brings a higher signal-to-noise ratio and a higher sensitivity for slow flow than traditional B-mode US (9). CE-US has been widely used in many fields including hepatocellular carcinomas (HCC), breast cancers, and colorectal cancers (10-12). Previous studies have evaluated the efficacy of chemotherapy in different cancers using CE-US, and reported that CE-US could be useful in evaluating tumor progression by detecting changes in tumor vascularity (10-12). Kudo et al. compared B-mode US with CE-US for the surveillance of HCC and recommended CE-US as a useful detection method for HCC (13).

The present study aims to explore whether CE-US is also superior to B-mode US in detecting early-stage PC. Therefore, we performed this retrospective study to compare B-mode US and CE-US for the surveillance of early-stage PC. We present the following article in accordance with the STARD reporting checklist (available at http://dx.doi.org/10.21037/jgo-20-412).

\section{Methods}

\section{Patient selection and data collection}

This was a retrospective study performed in the Affiliated Hospital of Jiangnan University. The data of patients who were admitted to our hospital between June 2015 and December 2019 were collected for further analysis. Patients were enrolled according to the following inclusion criterion: (I) aged between 18 and 80 years; (II) patients who were suspected of pancreatic insufficiency, pancreatic cyst, main pancreatic duct dilation, or elevation of some tumor marker, such as carcino-embryonic antigen (CEA), carbohydrate antigen (CA) 199, and CA125; or (III) patients who successively received B-mode US and CE-US examinations. Patients were excluded based on the following criteria: (I) they were previously diagnosed with PC; (II) they were previously diagnosed with acute or chronic pancreatitis; or (III) they were judged inappropriate for inclusion by the investigators.

Upon enrolment in this study, patients' demographic and clinical data were collected, including age, gender, tumor location, tumor size, and Tumor Node Metastasis (TNM) classification. The study was performed in compliance with the ethical principles of the Declaration of Helsinki (as revised in 2013), and was approved by the ethics committee of the Affiliated Hospital of Jiangnan University (No. 2020000045). Signed informed consent was not obtained due to the retrospective nature of the study.

\section{$B$-mode US examination}

An Aplio XV system (Toshiba Medical Systems, Tokyo, Japan) and a 375BT convex transducer with a frequency range of $1.9-6.0 \mathrm{MHz}$ were used in this study for conventional B-mode US examinations, which were performed by skilled radiologists. For conventional B-mode US examination, patients fasted for at least $8 \mathrm{~h}$ prior to the examination. The gallbladder and adjacent liver parenchyma were firstly examined using conventional grayscale US. The target lesion, including its position, size, shape, echogenicity, and number was then determined. Finally, blood flow within the lesion was evaluated by color 
Doppler US.

\section{CE-US examination}

An Aplio XV system and contrast harmonic imaging (mechanical index ranges from 0.08-0.10) were used for CE-US examinations, which were performed by skilled radiologists. SonoVue (Bracco, Milan, Italy) was used as the contrast agent in this study. For CE-US examination, $3 \mathrm{~mL}$ SonoVue was intravenously injected into the antecubital vein followed by $3 \mathrm{~mL}$ physiological saline. The target lesion was continuously observed in a harmonic mode for at least $3 \mathrm{~min}$ immediately following injection of the contrast agent. Target lesions were categorized into three groups according to their enhancement effects (compared with the surrounding tissues), including hypo-enhanced, hyperenhanced, and iso-enhanced groups. Hypo-enhancement was indicative of invasive adenocarcinoma (1).

\section{Confirmation of diagnosis}

For patients who did not receive surgical resection in our hospital, the diagnosis was confirmed by needle aspiration biopsy or endoscopic retrograde cholangiopancreatography (ERCP) following B-mode US and CE-US. However, detailed pathological examination was performed for patients who received surgical resection in our hospital, using surgical specimens to further confirm the diagnosis. Also, the size of the PC was determined by surgical specimens or ERCP.

\section{Statistical analysis}

Statistical analysis in this study was performed using SPSS software (version 23, IBM Corporation, NY, USA). Sensitivity, specificity, accuracy, positive predictive value (PPV), and negative predictive value (NPV) of B-mode US and CE-US were retrospectively calculated. Continuous data were expressed as the mean \pm standard deviation. Binary data were expressed as the number (percentage). The detection rate comparisons of B-mode US and CE-US were analyzed using the McNemar test. Receiver operating characteristic (ROC) curves were generated using SPSS. A subgroup analysis was performed to determine the effects of tumor size on the diagnostic accuracy of B-mode US and CE-US. A P value $<0.05$ was considered statistically significant.

\section{Results}

\section{Patient characteristics}

There were 3,542 patients admitted to the Affiliated Hospital of Jiangnan University for potential pancreatic insufficiency between June 2015 and December 2019. After retrospectively analyzing their data and examination results, we excluded 3,414 patients due to no specific lesions detected or huge lesions that had been diagnosed as PC. Finally, 128 patients who successively received B-mode US and CE-US examinations for possible PC were included in this study; a flow chart of the study is shown in Figure 1. According to the results of fine needle aspiration biopsy, ERCP, or surgical resection, 74 patients were diagnosed as PC and 54 patients were diagnosed with benign pancreatic lesions.

Demographic and clinical characteristics are listed in Table 1. There were 79 male patients and 49 female patients, with a mean age of $61.1 \pm 6.7$ years. Among the 74 PC patients, approximately $60 \%$ of the PCs were located at the head of pancreas. We enrolled early-stage PC patients in this study and TNM classifications of these patients are listed in Table 1 . Nearly half of the enrolled patients were diagnosed at T1cN0M0 (33, 44.6\%). The mean size of the PC was $17.8 \pm 4.9 \mathrm{~mm}$.

\section{Diagnostic capability}

Some representative US images of two patients are presented in Figure 2. The detectability of B-mode US and CE-US in PC and benign pancreatic lesions are listed in Table 2. Sixty-eight of the 74 PC patients were diagnosed correctly by CE-US, and surprisingly, all 54 patients with benign pancreatic lesions were also correctly diagnosed. However, B-mode US did not achieve a satisfying result; only 55 of the $74 \mathrm{PC}$ patients and 50 of the 54 patients with benign pancreatic lesions were correctly diagnosed. We calculated the sensitivity, specificity, accuracy, PPV, and NPV of B-mode US and CE-US (shown in Table 3). Our results indicate that CE-US outperformed B-mode US in each diagnostic capability parameter, especially specificity and PPV. According to the results of McNemar test, the diagnostic capability of CE-US was statistically superior to B-mode US $(\mathrm{P}=0.045)$. As shown in Figure 3, the ROC curve demonstrated that the area under the curve (AUC) of CE-US was 0.959, while the AUC of B-mode US was 0.835 . 


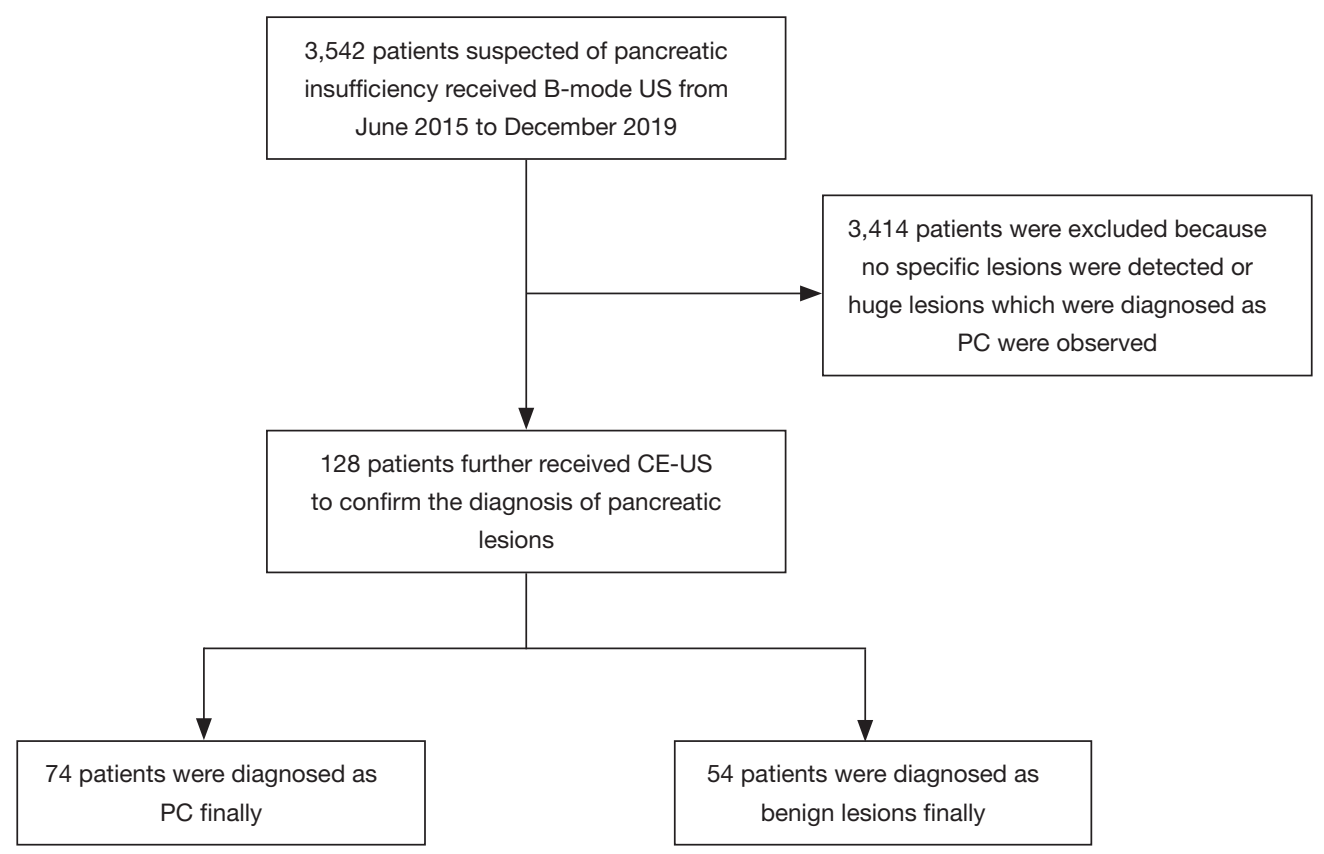

Figure 1 Flow chart of this study. US, ultrasonography; PC, pancreatic cancer; CE-US, contrast-enhanced ultrasonography.

Table 1 Patient characteristics

\begin{tabular}{|c|c|}
\hline Characteristics & Value \\
\hline Total & 128 \\
\hline Male/female & $79(61.7 \%) / 49$ (38.3\%) \\
\hline Mean age, years & $61.1 \pm 6.7$ \\
\hline \multicolumn{2}{|l|}{ Final diagnosis } \\
\hline Benign lesions & $54(42.2 \%)$ \\
\hline PC & $74(57.8 \%)$ \\
\hline \multicolumn{2}{|l|}{ Location of PC } \\
\hline Head & $45(60.8 \%)$ \\
\hline Body and tail & $29(39.2 \%)$ \\
\hline \multicolumn{2}{|c|}{ TNM classification of PC } \\
\hline TisNOMO & $3(4.1 \%)$ \\
\hline T1aN0M0 & $8(10.8 \%)$ \\
\hline T1bNOMO & $11(14.9 \%)$ \\
\hline T1cN0M0 & $33(44.6 \%)$ \\
\hline T2NOMO & $15(20.3 \%)$ \\
\hline T2N1M0 & $4(5.4 \%)$ \\
\hline Mean size of PC (mm) & $17.8 \pm 4.9$ \\
\hline
\end{tabular}

$\mathrm{PC}$, pancreatic cancer.

\section{Subgroup analysis}

Next, a subgroup analysis was performed to determine the effects of tumor size on the diagnostic accuracy of B-mode US and CE-US. We divided patients into four subgroups based on the tumor size (Table 4). The results indicated that B-mode US and CE-US exhibited similar capacities to diagnose relatively large tumors (PC size $>20 \mathrm{~mm}$, $\mathrm{P}=1.000$ ). However, for smaller tumors, CE-US exhibited better accuracy than $\mathrm{B}$-mode US (size $<20 \mathrm{~mm}, \mathrm{P}=0.002$; size $<10 \mathrm{~mm}, \mathrm{P}=0.043$; size $<5 \mathrm{~mm}, \mathrm{P}=0.025$ ).

\section{Discussion}

This retrospective study enrolling 128 patients suspected of pancreatic insufficiency compared the diagnostic capacity of B-mode US and CE-US for the surveillance of earlystage PC. To our knowledge, this is the first study in this field. Our study indicated that CE-US was superior to $\mathrm{B}$-mode US in detecting early-stage PC, especially smaller sized tumors. The results of this study provide evidence for the clinical application of CE-US in the detection of early-stage PC.

CE-US was first used to detect the characterization of liver tumors by Wilson et al. in 2000 (14). It was 

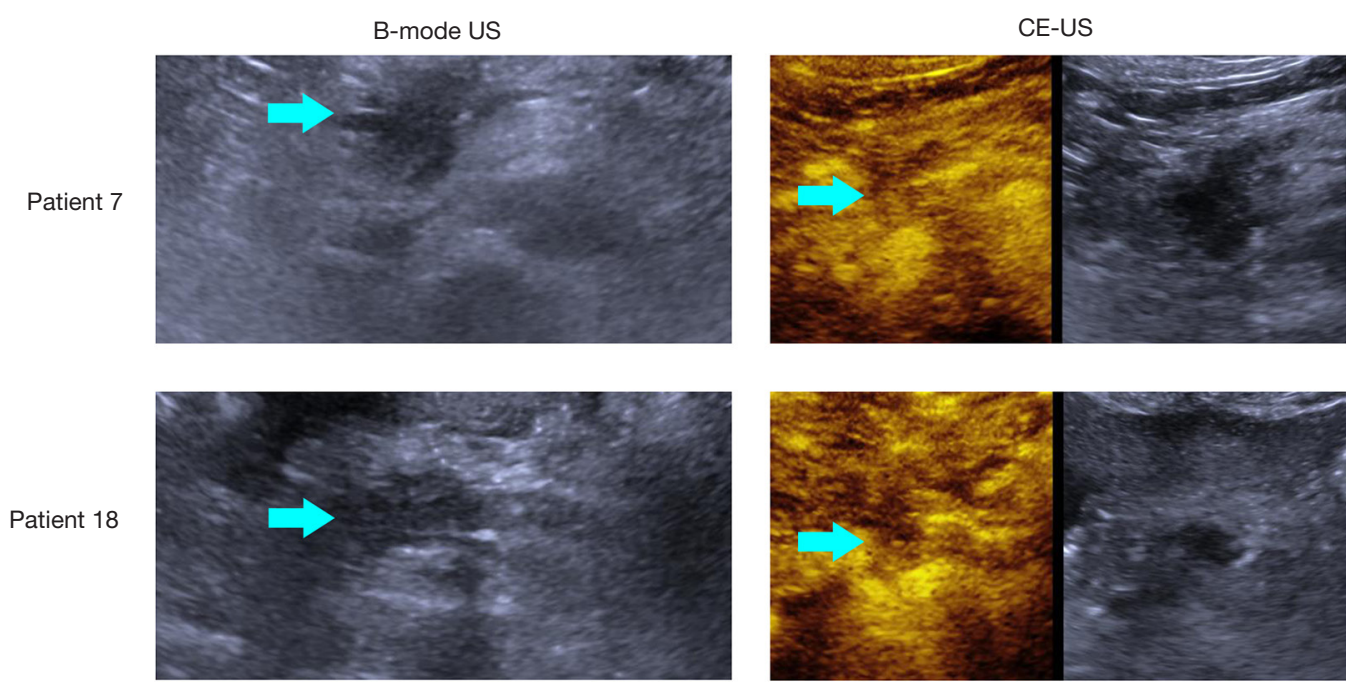

Figure 2 Representative B-mode US and CE-US images of two patients. US, ultrasonography; CE-US, contrast-enhanced ultrasonography. Arrow: the location of PC.

Table 2 Detectability of B-mode US and CE-US in PC and benign lesions

\begin{tabular}{lccccc}
\hline \multirow{2}{*}{ Detectability } & \multicolumn{3}{c}{ B-mode US } & & CE-US \\
\cline { 2 - 5 } & PC & Benign lesions & PC & Benign lesions \\
\hline Positive & 68 & 6 & 55 & 19 \\
Negative & 0 & 54 & 4 & 50 \\
\hline
\end{tabular}

US, ultrasonography; CE-US, contrast-enhanced ultrasonography; PC, pancreatic cancer.

Table 3 Diagnostic capability of B-mode US and CE-US in PC and benign lesions

\begin{tabular}{lcc}
\hline Characteristics & B-mode US & CE-US \\
\hline Sensitivity & $68 / 68(100 \%)$ & $55 / 59(93.2 \%)$ \\
specificity & $54 / 60(90 \%)$ & $50 / 69(72.5 \%)$ \\
Accuracy & $122 / 128(95.3 \%)$ & $105 / 128(82.0 \%)$ \\
PPV & $68 / 74(91.9 \%)$ & $55 / 74(74.3 \%)$ \\
NPV & $54 / 54(100 \%)$ & $50 / 54(92.6 \%)$ \\
- & - & - \\
\hline
\end{tabular}

US, ultrasonography; CE-US, contrast-enhanced ultrasonography; PC, pancreatic cancer.

also subsequently used in the detection of pancreatic lesions and was reported as an effective measurement for PC (15). The main limitation of CE-US is the use of contrast agents, which may cause anaphylaxis and some other severe adverse reactions. The contrast agent used in this study was SonoVue, and no significant adverse reactions were observed.
At present, CE-US has not been widely used for the detection of PC in China; B-mode US is the most used measurement for normal physical examinations. In our study, B-mode US could only provide a basic diagnosis, with a relatively high sensitivity of $93.2 \%$ and a relatively low specificity of $72.5 \%$ for PC. If a suspicious tumor is found, the patient will receive further examination, as was the 
case in our study, including contrast-enhanced computed tomography (CE-CT) or magnetic resonance imaging (MRI). CE-CT has been utilized in the diagnosis of PC longer than CE-US. However, the diagnostic capacity of CE-CT is not satisfying, especially in smaller sized PC; the sensitivity of CE-CT reportedly only ranges from $72-77 \%$ for PC that is smaller than $20 \mathrm{~mm}$ (16). Therefore, a more sensitive examination is still needed. D'Onofrio et al. reported in 2013 that CE-US was at least as sensitive as CE-CT in the diagnosis of PC (17). Moreover, Tanaka et al. recently reported that the sensitivity of CT-US (97\%) was significantly superior to that of CE-CT in the diagnosis of PC (77\%) (1). As for MRI, it remains to be one of standard techniques for the diagnosis of PC. However, Pezzilli et al. found that CE-US exhibited a similar

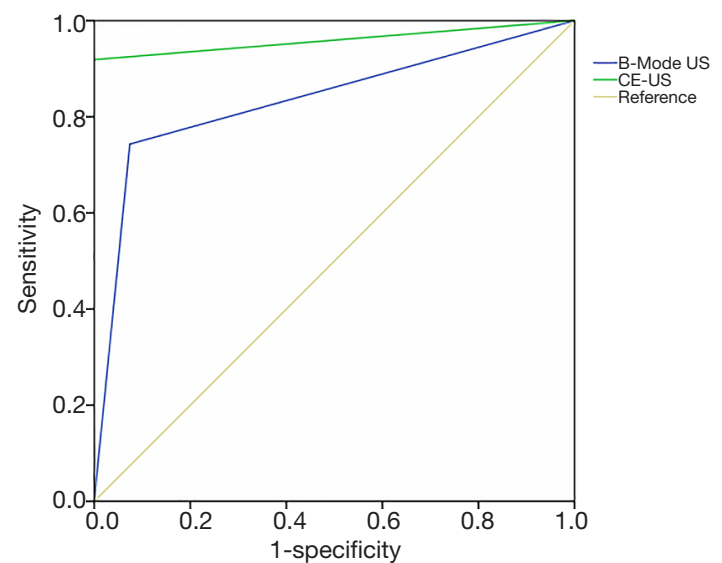

Figure 3 ROC curve analysis for B-mode US and CE-US in the diagnosis of PC. The AUC of CE-US and B-mode US were 0.959 and 0.835 , respectively. US, ultrasonography; CE-US, contrastenhanced ultrasonography; ROC, receiver operating characteristic; AUC, area under the curve; PC, pancreatic cancer. sensitivity and specificity to MRI in evaluating pancreatic lesions greater than $2 \mathrm{~cm}$ or smaller than $1 \mathrm{~cm}(18)$.

Considering the associated time and costs, CE-US could provide a better option than CE-CT and MRI to some degree. Moreover, CE-US offers other advantages over CE-CT or MRI in the evaluation of PC. Firstly, real-time dynamic imaging in CE-US makes it possible to visualize the enhancement pattern of lesions. Also, CE-US provides increased vascular permeability to characterize malignant tumors with the use of a contrast agent. Furthermore, the operation and parameters can be adjusted at any time during the CE-US procedure according to the observed enhancement pattern of lesions in order to obtain satisfactory results. Lastly, contrast agents used in CE-US are safer than those used in contrast-enhanced CT, and can be used in patients with renal insufficiency (19).

However, CE-US also has some disadvantages compared to other examinations. Most importantly, the performance of CE-US is highly dependent on the experience of operators and the cooperation of patients (20). In addition, satisfying results may not be achieved in obese patients using CE-US, especially in obese patients with deep lesions (21).

There were some limitations in this study to be noted. Firstly, this was a retrospective study, and thus some bias may be inevitable due to the nature of the study. Secondly, the size of $\mathrm{PC}$ was measured after surgical resection in most studies. However, some patients did not receive surgical resection in our hospital; $\mathrm{PC}$ size in these patients was measured by CE-US, which may generate some bias in the results. Thirdly, PC undetectable with conventional B-mode US was not included in this study. As is known, the visibility of the pancreas is reduced in some obese patients, which may also result in selection bias.

In conclusion, this study confirmed the diagnostic

Table 4 Comparison of the accuracy of B-mode US and CE-US for different sizes of PC

\begin{tabular}{|c|c|c|c|c|}
\hline Characteristics & Number & Accuracy of B-mode US & Accuracy of CE-US & $P$ value \\
\hline \multicolumn{5}{|l|}{ Size, $\mathrm{mm}$} \\
\hline$\geq 20$ & 19 & $18 / 19(94.7 \%)$ & $19 / 19(100 \%)$ & 1.000 \\
\hline$<20$ & 55 & $37 / 55(67.3 \%)$ & 49/55 (89.1\%) & 0.002 \\
\hline$<5$ & 11 & $3 / 11(27.3 \%)$ & $7 / 11(63.6 \%)$ & 0.025 \\
\hline
\end{tabular}

US, ultrasonography; CE-US, contrast-enhanced ultrasonography; PC, pancreatic cancer. 
capacity of CE-US for early PC. Considering that there are currently few specific serological biomarkers for PC, CEUS could act as an important supplementary examination method after B-mode US screening. Moreover, smaller sized tumors (less than $20 \mathrm{~mm}, 10 \mathrm{~mm}$, or even $5 \mathrm{~mm}$ ) could be clearly distinguished by CE-US.

\section{Acknowledgments}

Funding: None.

\section{Footnote}

Reporting Checklist: The authors have completed the STARD reporting checklist. Available at http://dx.doi.org/10.21037/ jgo-20-412

Data Sharing Statement: Available at http://dx.doi. org/10.21037/jgo-20-412

Conflicts of Interest: All authors have completed the ICMJE uniform disclosure form (available at http://dx.doi. org/10.21037/jgo-20-412). The authors have no conflicts of interest to declare.

Ethical Statement: The authors are accountable for all aspects of the work in ensuring that questions related to the accuracy or integrity of any part of the work are appropriately investigated and resolved. The study was performed in compliance with the ethical principles of the Declaration of Helsinki (as revised in 2013), and was approved by the ethics committee of the Affiliated Hospital of Jiangnan University (No. 2020000045). Signed informed consent was not obtained due to the retrospective nature of the study.

Open Access Statement: This is an Open Access article distributed in accordance with the Creative Commons Attribution-NonCommercial-NoDerivs 4.0 International License (CC BY-NC-ND 4.0), which permits the noncommercial replication and distribution of the article with the strict proviso that no changes or edits are made and the original work is properly cited (including links to both the formal publication through the relevant DOI and the license). See: https://creativecommons.org/licenses/by-nc-nd/4.0/.

\section{References}

1. Tanaka S, Fukuda J, Nakao M, et al. Effectiveness of Contrast-Enhanced Ultrasonography for the Characterization of Small and Early Stage Pancreatic Adenocarcinoma. Ultrasound Med Biol 2020;46:2245-53.

2. Lee ES, Lee JM. Imaging diagnosis of pancreatic cancer: a state-of-the-art review. World J Gastroenterol 2014;20:7864-77.

3. Ikuta S, Sonoda T, Aihara T, et al. A combination of platelet-to-lymphocyte ratio and carbohydrate antigen 19-9 predict early recurrence after resection of pancreatic ductal adenocarcinoma. Ann Transl Med 2019;7:461.

4. Abbassi R, Algül H. Palliative chemotherapy in pancreatic cancer-treatment sequences. Transl Gastroenterol Hepatol 2019;4:56.

5. Leem G, Chung MJ, Park JY, et al. Clinical Value of Contrast-Enhanced Harmonic Endoscopic Ultrasonography in the Differential Diagnosis of Pancreatic and Gallbladder Masses. Clin Endosc 2018;51:80-8.

6. Wang L, Gaddam S, Wang N, et al. Multiparametric Mapping Magnetic Resonance Imaging of Pancreatic Disease. Front Physiol 2020;11:8.

7. Ciofoaia V, Haddad NG, Smith JP. How many pancreatic cysts are out there and how to best manage them? Transl Cancer Res 2018;7:S500-5.

8. Kuroki-Suzuki S, Kuroki Y, Nasu K, et al. Pancreatic cancer screening employing noncontrast magnetic resonance imaging combined with ultrasonography. Jpn J Radiol 2011;29:265-71.

9. Rafailidis V, Huang DY, Yusuf GT, et al. General principles and overview of vascular contrast-enhanced ultrasonography. Ultrasonography 2020;39:22-42.

10. Fukuda H, Numata K, Hara K, et al. Comparison of vascularity observed using contrast-enhanced $3 \mathrm{D}$ ultrasonography and pathological changes in patients with hepatocellular carcinoma after sorafenib treatment. J Cancer 2018;9:2408-14.

11. Wu X, Tang L, Huang W, et al. Contrast-enhanced ultrasonography and blue dye methods in detection of sentinel lymph nodes following neoadjuvant chemotherapy in initially node positive breast cancer. Arch Gynecol Obstet 2020;302:685-92.

12. Amadori M, Barone D, Scarpi E, et al. Dynamic contrastenhanced ultrasonography (D-CEUS) for the early 
prediction of bevacizumab efficacy in patients with metastatic colorectal cancer. Eur Radiol 2018;28:2969-78.

13. Kudo M, Ueshima K, Osaki Y, et al. B-Mode Ultrasonography versus Contrast-Enhanced Ultrasonography for Surveillance of Hepatocellular Carcinoma: A Prospective Multicenter Randomized Controlled Trial. Liver Cancer 2019;8:271-80.

14. Wilson SR, Burns PN, Muradali D, et al. Harmonic hepatic US with microbubble contrast agent: initial experience showing improved characterization of hemangioma, hepatocellular carcinoma, and metastasis. Radiology 2000;215:153-61.

15. Takeshima K, Kumada T, Toyoda H, et al. Comparison of IV contrast-enhanced sonography and histopathology of pancreatic cancer. AJR Am J Roentgenol 2005;185:1193-200.

16. Yoon SH, Lee JM, Cho JY, et al. Small ( $\leq 20 \mathrm{~mm})$ pancreatic adenocarcinomas: analysis of enhancement patterns and secondary signs with multiphasic multidetector CT. Radiology 2011;259:442-52.

17. D'Onofrio M, Crosara S, Signorini M, et al. Comparison between CT and CEUS in the diagnosis of pancreatic

Cite this article as: $\mathrm{Xu} \mathrm{J}$, Zhang M, Cheng G. Comparison between B-mode ultrasonography and contrast-enhanced ultrasonography for the surveillance of early stage pancreatic cancer: a retrospective study. J Gastrointest Oncol 2020;11(5):10901097. doi: 10.21037/jgo-20-412 adenocarcinoma. Ultraschall Med 2013;34:377-81.

18. Pezzilli R, Serra C, Calculli L, et al. Three-dimensional contrast-enhanced ultrasonography of intraductal papillary mucinous neoplasms of the pancreas: a comparison with magnetic resonance imaging. Pancreas 2013;42:1164-8.

19. Piscaglia F, Bolondi L; Italian Society for Ultrasound in Medicine and Biology (SIUMB) Study Group on Ultrasound Contrast Agents. The safety of Sonovue in abdominal applications: retrospective analysis of 23188 investigations. Ultrasound Med Biol 2006;32:1369-75.

20. Ryu SW, Bok GH, Jang JY, et al. Clinically useful diagnostic tool of contrast enhanced ultrasonography for focal liver masses: comparison to computed tomography and magnetic resonance imaging. Gut Liver 2014;8:292-7.

21. Muskiet MHA, Emanuel AL, Smits MM, et al. Assessment of real-time and quantitative changes in renal hemodynamics in healthy overweight males: Contrastenhanced ultrasonography vs para-aminohippuric acid clearance. Microcirculation 2019;26:e12580.

(English Language Editor: A. Kassem) 